\title{
Ear, Nose and Throat Disorders in Renal Patients
}

\author{
Kamel El Reshaid* \\ Department of Medicine, Faculty of Medicine, Kuwait University, Kuwait
}

*Corresponding author: Kamel El Reshaid, Department of Medicine, Faculty of Medicine, Kuwait University, Kuwait

\begin{abstract}
Patients with renal disease can present with acute renal failure (RF), chronic RF, and end-stage renal disease. Specific otolaryngologic disorders can associate the underlying etiology of acute renal diseases such as infections and autoimmune diseases or its drug therapy. Moreover, chronic RF is associated with hypertension, uremia, renal osteodystrophy and platelets dysfunction. Renal replacement therapy has inherent risk of anticoagulation with hemodialysis and immunosuppression with kidney transplantation.
\end{abstract}

Keywords: Autoimmune Disease; ENT; Drug Side Effect; Renal Failure

\section{Introduction}

In the United States acute renal failure (RF) affects about 3 per 1,000 people a year [1]. Chronic RF affects about 1 in 1,000 people with 3 per 10,000 people newly develop the condition each year [2]. Patients with RF can present with acute RF, chronic RF, and endstage renal disease (ESRD) [3]. The latter is severe, irreversible and advanced kidney disease. Patients with acute RF can be managed conservatively with

a. elimination of the offending factor such as dehydration, drug-allergy (acute interstitial nephritis), infection/ autoimmune disease and obstruction of urinary tract and

b. correction of complications of RF viz. uremia, hyperkalemia and fluid overload and their drug-therapy. If already have severe RF; temporary (supportive) dialysis is done till recovery [4].

On the other hands; chronic RF is due to hereditary disorders such as Alport's and polycystic kidney disease as well as primary and secondary glomerulopathy (GN), previous reflux nephropathy (chronic pyelonephritis), hypertension and neglected obstruction. Those patients are managed with

a. control of the underlying disease to prolong kidney survival,

b. control of co-morbid conditions associated with RF viz. hypertension, hyperkalemia, fluid overload, renal osteodystrophy and anemia c. decrease intraglomerular pressure to decrease glomerulosclerosis, to prolong kidney survival, with angiotensin converting enzyme inhibitors/angiotensin receptors blockers (ACEI/ARB) and low-protein diet, and

d. renal replacement therapy (RRT) with maintenance dialysis and subsequently kidney transplantation if they had reached ESRD [5].

Kidney transplantation is done, if ESRD patients are fit for it and already have donors. The main problems with RRT are

a. anticoagulation, usually with heparin which lasts for 4-6 hours post hemodialysis session and

b. immunosuppressive side-effects of agents used to prevent rejection in the transplanted kidney.

Immunosuppression is usually heavy in the first 3-months and can be associated with bacterial infections while viral, fungal and parasitic opportunistic infections and certain malignancies can develop later [6].

\section{Specific Reno/Medical Causes of Otolaryngologic Disorders}

Ear

a) Tinnitus: Hypertension and uremia.

b) Vertigo: Hypertensive cerebella/pontine lacunar infarct. 
c) Drowsiness: Dehydration, hypo/hyperglycemia electrolytes disturbances especially hyponatremia, hypercalcemia, diabetic neuropathy, drugs viz. Aldose (+ postural hypotension), sedatives or Lyrica, anticonvulsants,

d) Deafness: Sensori-neural deafness with Alport's and drug induced viz. high-dose Lasix and Aminoglycosides.

e) Trigeminal Neuralgia: Can be mistaken for local ear/ teeth disorder or CVA/migraine.

f) TB of middle and inner ear and herpes zoster, candida and pyogenic otitis externa in immunocompromised patients.

\section{Throat}

a) Pharyngitis: Epstein-Barr virus (EBV) infection can present with prolonged fever, coated tonsils, lymphadenopathy, weight loss. Moreover, mouth ulcers are common in patients with systemic lupus erythematosus (SLE), Bechet's disease and GERD. Oral and esophageal candidiasis (thrush) is common following steroid-therapy and mouth ulcers after Mycophenolate and Methotrexate treatment. Mycophenolate is notorious in induction of fever, dyspnea, mouth ulcers and diarrhea due to activation of Cytomegalovirus infection (CMV). URTI can lead to post-strep GN (a self-limited hypertension, proteinuria and hematuria). A similar yet recurrent pattern can be seen coinciding with URTI due to IgA nephropathy (spheritic GN). Throat pain with lymphoid hyperplasia is a common side-effect of ACEI and rarely with ARB.

b) Dry Mouth: Sicca syndrome (Sjogren's). Drugs viz. Calcitriol, Clonidine, Codeine, anti-emetic, antihistaminics, antidepressants, diuretics (Amelioride, HCTZ, Torosemide), Omeperazole, Prazocin, Physio tens and Tramadol.

c) Gingival Hyperplasia: usually due to drugs viz. Phenytoin, Cyclosporine A, Calcium channel blockers

d) Renal osteodystrophy: rarely affects teeth yet bony cavitary lesions (Brown tumors) and ostemalacia with poor bone healing if patients are subjected to ENT surgery.

e) Hoarseness: GERD, ACEI, angioneurotic edema, or vocal cord palsy after thyroid/parathyroid surgery. Also; diseases such as hypothyroidism, multiple sclerosis and myasthenia gravis.

\section{Nose}

a) Epistaxis: Hypertension, moderate renal disease + platelets dysfunction, thrombocytopenia (SLE, myeloproliferative diseases, drug induced, EBV, CMV) and excessive heparin during hemodialysis/anti-platelet and anticoagulants.

b) Nasal/paranasal destructive lesions: in Granulomatosis with polyangiitis (Wegner's vasculitis) which manifests with nasal/ paranasal/middle and inner ear/pulmonary disease. c) Allergic rhinitis and asthma-like: Hypersensitivity angrites (Churg-Strauss vasculitis) and drug-induced (NSAIDs).

\section{Most Important Message}

a) Avoid NSAIDs in treating patients with renal disease [7].

b) Avoid dye study without consulting a nephrologist [8].

c) Adjust dose of some antibiotic.

d) Renal patients have premature atherosclerosis: CVA, renovascular hypertension, pre-mature hearing loss [9].

e) SLE and vasculitis are a multi-system autoimmune disease with hematological disorders and thrombotic potentials as well as associated with multiple side-effects of medications viz. corticosteroids and immunosuppressive agents. The latter can predispose to life threatening viral, bacterial and fungal infections as well as malignancies [10].

f) Reactivation of latent $\mathrm{TB}$ is common in dialysis and transplant patients since immunocompromised.

\section{Conclusion}

OL manifestations in kidney patients; may be the tip of the iceberg of multiple reno/medical disorders and can aid in and their management.

\section{References}

1. Ferri Fred F (2017) Ferri's Clinical Adviser 2018 E-Book: 5 Books in 1. Elsevier Health Sciences, pp. 37.

2. Ferri Fred F (2017) Ferri's Clinical Adviser 2018 E-Book: 5 Books in 1 Elsevier Health Sciences, pp. 294.

3. Levey AS, Levin A, Kellum JA (2013) Definition and classification of kidney diseases. Am J Kidney Dis 61: 686-688.

4. (2012) KDIGO Clinical Practice Guideline for Acute Kidney Injury. Kidney Int Suppl 2: 8.

5. (2012) KDIGO Clinical Practice Guideline for the Evaluation and Management of Chronic Kidney Disease. Kidney Int Suppl 3: 5.

6. (2013) United States Renal Data System. USRDS 2013 Annual Data Report: Atlas of Chronic Kidney Disease and End-Stage. Renal Disease in the United States. National Institutes of Health; National Institute of Diabetes and Digestive and Kidney Diseases, Bethesda.

7. El Reshaid K, Kapoor M, Johny KV, Sugathan TN (1993) Acute renal failure in Kuwait, a prospective study. J Trop Med Hygiene 96: 323-329.

8. Kamel El Reshaid, Shaikha Al Bader (2019) Prevention of contrastinduced nephropathy by 16-hours post-contrast CVVHD. IJIRMS 4: 318321.

9. Shoji T, Emoto M, Tabata T, Kimoto E, Shinohara K, et al. (2002) Advanced atherosclerosis in predialysis patients with chronic renal failure. Kidney Int 61: 2187-2192.

10. British Thoracic Society Standards of Care Committee and Joint Tuberculosis Committee, Milburn H, Ashman N, Davies P, Doffman S, et al. (2010) Guidelines for the prevention and management of Mycobacterium tuberculosis infection and disease in adult patients with chronic kidney disease. Thorax 65: 557-570. 
(C) (1) This work is licensed under Creative

To Submit Your Article Click Here: Submit Article

DOI: $10.32474 /$ SJO.2019.02.000143

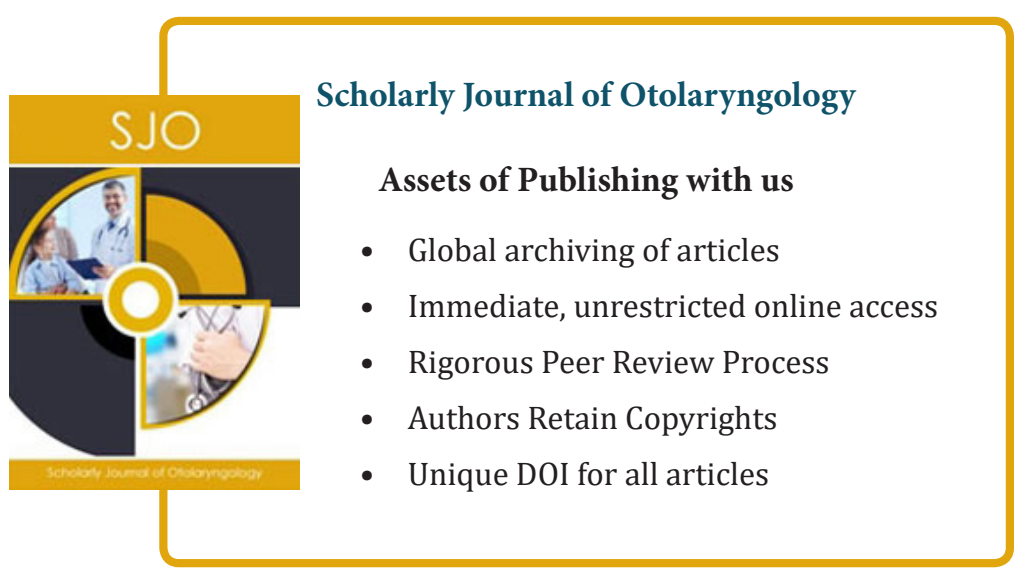

